\title{
Amoxicillin rash in patients with infectious mononucleosis: evidence of true drug sensitization
}

\author{
Katinka Ónodi-Nagy ${ }^{1 *}$, Ágnes Kinyó ${ }^{1}$, Angéla Meszes ${ }^{1}$, Edina Garaczi ${ }^{1}$, Lajos Kemény ${ }^{1,2}$ \\ and Zsuzsanna Bata-Csörgő ${ }^{1,2}$
}

\begin{abstract}
Background: It hasn't been clearly understood yet whether sensitization to antibiotics, the virus itself or transient loss of drug tolerance due to the virus, is responsible for the development of maculopapular exanthems following amoxicillin intake in patients with infectious mononucleosis. We aimed to examine whether sensitization to penicillin developed among patients with skin rash following amoxicillin treatment within infectious mononucleosis.

Methods: Ten patients were investigated for drug sensitization by lymphocyte transformation test and six patients were further tested by prick-, intradermal and patch tests employing the penicillin's main antigens.

Results: Lymphocyte transformation test showed negative results with amoxicillin, while one patient had positive reaction to cefixime. Six patients with suspected sensitization to amoxicillin were then investigated by in vivo tests. Prick tests were negative in all six patients, but the intradermal tests showed positive reactions in four patients.

Conclusions: Our data demonstrate that in vitro testing is not sensitive enough in determining drug sensitization to penicillin. In vivo tests should be performed to detect sensitization and indeed with skin tests our results confirmed that sensitization to aminopenicillin may develop within infectious mononucleosis.
\end{abstract}

Keywords: Amoxicillin, Sensitization to antibiotics, Infectious mononucleosis, Drug tests

\section{Introduction}

Infectious mononucleosis (IM) is an acute disease mostly caused by a widespread human $\gamma$-herpes virus, the Epstein-Barr virus (EBV) or a human $\beta$-herpes virus, the cytomegalovirus. The primary infection appears predominantly in children, adolescents and young adults [1]. Symptoms start with a prodromal phase including subfebrility, malaise, arthralgia and myalgia, like any common upper respiratory tract infection [2]. The classic features, fever, tonsillopharyngitis, lymphadenopathy, leukocytosis and hepatosplenomegaly, are helpful in differentiation from bacterial infection. Skin eruptions may develop during the infection. These eruptions are maculopapular exanthems, morbilliform eruptions on the whole body, in severe cases the progressive skin reaction turns into erythroderma (Figure 1). A severe cutaneous reaction such as erythema multiforme is exceedingly rare, although

\footnotetext{
* Correspondence: onodikatinka@gmail.com

'Department of Dermatology and Allergology, University of Szeged, Albert Szent-Györgyi Medical Center, Korányi fasor 6, Szeged 6720, Hungary Full list of author information is available at the end of the article
}

possible manifestation [3]. The skin symptoms may develop due to the viral infection, however, these patients often use antibiotics and it is also well-known that viral infections enhance the risk of drug allergic reactions $[4,5]$.

Eosinophil rich maculopapular exanthems occurring in mononucleosis rash are considered to be delayed type hypersensitivity reactions, in which Th2 T cells are activated and secrete IL-4, IL-5 and IL-13 that leads to eosinophilic inflammation. However secretion of IgE and IgG4 by B-cells accompanies the reaction, connecting the delayed reaction to immediate type I reaction [6]. We aimed to examine whether sensitization to penicillin developed among patients with cutaneous rash following amoxicillin treatment within IM.

\section{Findings \\ Materials and methods \\ Patient selection}

At the Department of Dermatology and Allergology, University of Szeged in Hungary among those patients who were treated between 2002 and 2012, ten young 


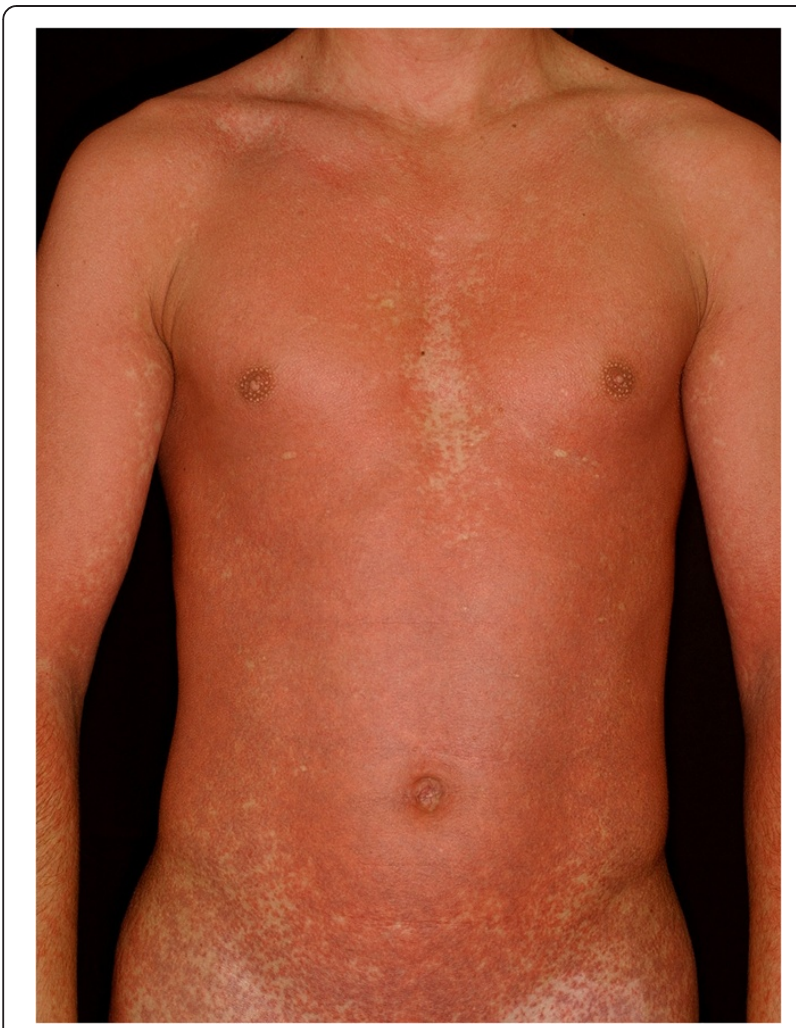

Figure 1 Amoxicillin rash in a patient with infectious mononucleosis (patient 4). The cutaneous eruptions developed a few days after the initiation of the antibiotic therapy. In severe cases the progressive maculopapular exanthems turn into erythroderma.

adults ( 5 men and 5 women, median age 22.9 , range $15-$ 35 years) with the diagnosis of IM, confirmed by EBV serological assay (specific IgM and IgG antibodies), associated with generalized maculopapular eruptions were examined for sensitization to antibiotics. All of these patients underwent antibiotic therapy prior to the appearance of skin eruptions. In all cases the antibiotic was amoxicillin/clavulanic acid, in 2 cases in addition to penicillin the patients were given clarithromycin or cefixime as well. Although clinically the skin symptoms of Drug Reaction with Eosinophilia and Systemic Symptoms (DRESS) can be indistinguishable, DRESS have strict criteria, which were not met in our patients [7].

\section{In vitro tests: lymphocyte transformation test (LTT)}

We examined mononucleosis infectiosa patients with a history of penicillin intake, with an in vitro method, the LTT, 1-1.5 months after the cessation of skin eruptions. The LTT was performed to determine T-cell proliferation as an indicator of drug sensitization as described previously [8,9], with minor modifications. Briefly, peripheral blood mononuclear cells were isolated from heparinized peripheral blood and cultured under defined conditions with various concentrations of the suspected drugs $(100 \mu \mathrm{g} / \mathrm{ml}$ and $10 \mu \mathrm{g} / \mathrm{ml}$ dilutions), in our cases with amoxicillin, amoxicillin/clavulanic acid, penicillin and cefixime [10]. We evaluated cell growth in the cultures. Cell growth was measured by using a colorimetric assay and an automatic microplate scanning spectrophotometer. The assay depends on the reduction of tetrazolium salt (MTT: 3-(4,5-dimethylthiazol-2-yl)-2,5-diphenyltetrazolium bromide) by living cells, to form a blue insoluble formazan product [11]. During the investigation we used the spontaneous cell growth as negative control, while the phytohaemagglutinin-stimulated cell culture served as positive control. The results were recognized as positive, if the drug stimulated cell numbers were at least twice higher that the negative control's (stimulation index $>2$ ).

\section{In vivo test: skin tests}

We performed in vivo cutaneous tests using penicillins in patients with negative LTT to amoxicillin. The remaining patients refused to consent to testing. Prick, intradermal and patch tests were performed using penicillin's main antigens: major determinant benzylpenicilloyl poly-L-lysine (PPL), minor determinant mix sodium benzylpenicillin, benzylpenicilloic acid, sodium benzylpenicilloate (MDM) from Diater Laboratorios (Penicillin allergenic determinants (DAP) ${ }^{\circ}$ test) $[12,13]$. We followed the investigation protocol given by the manufacturer [14]. Cutaneous tests were started with major determinants (Figure 2). If the prick tests at different dilutions were negative, the testing was continued with intradermal and then patch tests. Each prick and intradermal tests were read once 20 minutes elapsed since their application. Tests results were also read at 24, 48, 72 and 96 hours for detecting delayed reactions. Patch tests were performed using the powdered culprit drug mixed into vaseline (1:1). Allergens were applied to the upper back in individual round chambers (Curatest ${ }^{\circ}$, Spiromed Ltd.). Readings were performed at 48, 72, 96 hours and 7 days $[15,16]$. Although skin rashes occurring in mononucleosis are likely delayed type reactions, we performed immediate reading, because clinical history cannot always be trusted, patients will report a delayed reaction which is in fact an immediate one.

\section{Results}

Among those patients who were treated at our clinic between 2002 and 201210 patients (median age 22.9, range 15-35 years) with IM and maculopapular rash were examined by drug allergy tests. All patients took antibiotics before the appearance of skin symptoms. In all ten patients in vitro LTTs were performed with the suspected drugs. Amoxicillin/clavulanic acid enhanced drug-specific response in none of the cases. Increased lymphocyte proliferation was found with one peripheral blood sample after incubation with cefixime. Six out of 


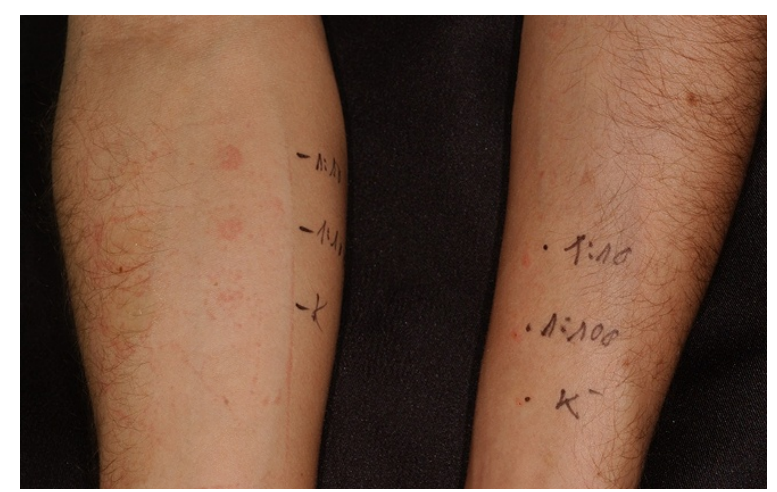

Figure 2 Positive cutaneous response (Penicillin allergenic determinants (DAP) ${ }^{\circledR}$ test). The in vivo cutaneous investigation was continued with intradermal testing, if the prick tests resulted in negative response at different dilutions. Skin tests were performed using penicillin's main antigens, major determinants and (PPL) and minor determinant mix (MDM). Cutaneous tests were started with major determinants, the negative control was saline solution. In this case we recognized positive skin reaction to MDM at 1:100 and 1:10 dilutions, which verified the development of sensitization (patient 4).

the 10 patients with suspected sensitization to amoxicillin and negative LTT results were further investigated in vivo by prick, intracutaneous and patch testing. All six patients showed negative responses with prick tests. The intradermal tests resulted in positive reactions in four subjects. Patch tests were performed after negative prick and intracutaneous testing with negative results in the remaining two patients (Table 1).

It is important to notice that the in vivo investigations were carried out at least six month after the disappearance of the eruptions which lead us to think that drug sensitization developed instead of a transient loss of tolerance, a transient Th-1 lymphocyte-mediated delayed type hypersensitivity reaction to the medication as discussed in the literature $[17,18]$.

\section{Discussion}

The development of skin rash following amoxicillin intake in patients with IM is quite frequent among beta-lactaminduced adverse drug reactions [19]. These eruptions are maculopapular exanthems. The exact mechanism behind them is unclear. It is not well explained yet, whether a true allergic drug reaction, virus-dependent rash or transient loss of drug tolerance due to the virus is responsible for the symptoms. The rash may be due to the viral infection itself, the incidence of skin eruption development in acute IM is $4.2-13 \%$ without drug intake, but often these patients are put on antibiotics, frequently amoxicillin, and the rash appears a few days after the initiation of the antibiotic therapy [20]. Following amoxicillin intake within acute IM the incidence of skin reactions ranges between $27.8 \%$ and $69 \%$, while in children, morbilliform skin eruptions nearly always develop following amoxicillin intake within acute IM $[4,21,22]$.

Our aim was to find out whether true amoxicillin sensitization developed for aminopenicillin among our patients. Evidence shows in recently published literature that the development of allergic reaction for aminopenicillin during a florid viral infection is definitely more prevalent as it was believed previously [4,5]. Although Renn et al. earlier demonstrated true sensitizations to amoxicillin in three patients with IM and clear history of amoxicillin intake with positive proliferative responses, we further investigated this phenomenon to provide more evidence. Our results add additional evidence that indeed in such patients drug sensitization develops during the infection. According to the current recommendations in drug allergy, positive skin tests for beta-lactam antiobiotics

Table 1 True sensitization to amoxicillin examined by in vivo cutaneous tests

\begin{tabular}{|c|c|c|c|c|c|c|c|}
\hline Patient & $\begin{array}{c}\text { Age } \\
\text { (years) }\end{array}$ & Gender & Culprit drug & LTT results & $\begin{array}{l}\text { Prick test } \\
\text { results }\end{array}$ & Intradermal test results & $\begin{array}{l}\text { Patch test } \\
\text { results }\end{array}$ \\
\hline 1 & 15 & female & amoxicillin/clavulanic acid & Negative & Negative & PPL 1:100 and 1:10 Positive & Not performed \\
\hline 2 & 19 & female & amoxicillin & Negative & Negative & MDM undiluted Positive & Not performed \\
\hline 3 & 29 & female & amoxicillin/clavulanic acid & Negative & Negative & PPL 1:10 Positive & Not performed \\
\hline 4 & 23 & male & amoxicillin/clavulanic acid & Negative & Negative & MDM 1:100 and 1:10 Positive & Not performed \\
\hline 5 & 35 & male & amoxicillin/clavulanic acid & Negative & Negative & Negative & Negative \\
\hline 6 & 24 & female & amoxicillin/clavulanic acid & Negative & Negative & Negative & Negative \\
\hline 7 & 21 & male & amoxicillin & Negative & Not performed & Not performed & Not performed \\
\hline 8 & 20 & female & amoxicillin/clavulanic acid & Negative & Not performed & Not performed & Not performed \\
\hline 9 & 16 & male & amoxicillin/clavulanic acid & Negative & Not performed & Not performed & Not performed \\
\hline 10 & 27 & male & amoxicillin/clavulanic acid; cefixime & Positive: cefixime & Not performed & Not performed & Not performed \\
\hline
\end{tabular}

PPL: major determinant: benzylpenicilloyl poly-L-lysine.

MDM: minor determinant mix: sodium benzylpenicillin, benzylpenicilloic acid, sodium benzylpenicilloate.

Bold text: Verified sensitization to penicillin.

Tests were done in the following chronology: LTT $\rightarrow$ Prick test (non-diluted PPL) $\rightarrow$ Intradermal test (1:100 dilution of PPL, 1:10 dilution of PPL, non-diluted PPL) $\rightarrow$ Prick test (non-diluted $M D M) \rightarrow$ Intradermal test (1:100 dilution of MDM, 1:10 dilution of MDM, non-diluted MDM) $\rightarrow$ Patch test (culprit drug). 
(following standardized reading) do not require challenge tests, to confirm clinical relevance. Skin tests are validated, while LTT is not. We can not explain the negative results of LTT in all of these cases to penicillins. The two patients with negative in vitro and in vivo test results need to be further investigated by performing cutaneous tests with the culprit drug and if this was negative a drug provocation test should be applied in order to prove that the patient did not developed penicillin allergy. In this work our primary aim was to demonstrate that true sensitization can occur within mononucleosis infectiosa patients suffering from amoxicillin rash.

With this investigation we would like to further emphasize the importance of allergy examination in patients with generalized skin lesions after penicillin intake in IM, to verify whether true sensitization developed.

\section{Conclusions}

Our data demonstrate that in vitro testing is not sensitive enough in determining drug sensitization for penicillin in patients who develop skin symptoms during mononucleosis infection. In vivo tests should be performed to detect sensitization and indeed with skin tests our results confirmed that sensitization to aminopenicillin may develop within infectious mononucleosis.

\section{Abbreviations \\ IM: Infectious mononucleosis; EBV: Epstein-barr virus; DRESS: Drug reaction with eosinophilia and systemic symptoms; LTT: Lymphocyte transformation test; MTT: 3-(4,5-dimethylthiazol-2-yl)-2,5-diphenyltetrazolium bromide; PPL: Benzylpenicilloyl poly-L-lysine; MDM: Minor determinant mix; DAP $\bullet$ : Penicillin allergenic determinants.}

\section{Competing interests}

The authors declare that they have no competing interest.

\section{Authors' contributions}

All authors read and approved the final manuscript.

\section{Acknowledgments}

We thank Andrea Gyimesi for her assistance in the preparation of the manuscript. The work was supported by TÁMOP - 4.2.2.A-11/1/KONV-2012-0035 project.

\section{Author details}

${ }^{1}$ Department of Dermatology and Allergology, University of Szeged, Albert Szent-Györgyi Medical Center, Korányi fasor 6, Szeged 6720, Hungary. ${ }^{2}$ Dermatological Research Group of the Hungarian Academy of Sciences, University of Szeged, Szeged, Hungary.

Received: 1 April 2014 Accepted: 8 October 2014

Published: 9 January 2015

\section{References}

1. Chovel-Sella A, Ben Tov A, Lahav E, Mor O, Rudich H, Paret G, et al. Incidence of rash after amoxicillin treatment in children with infectious mononucleosis. Pediatrics. 2013;131:e1424-7.

2. Leung AK, Rafaat M. Eruption associated with amoxicillin in a patient with infectious mononucleosis. Int J Dermatol. 2003:42:553-5.

3. Gonzalez-Delgado P, Blanes M, Soriano V, Montoro D, Loeda C, Niveiro E. Erythema multiforme to amoxicillin with concurrent infection by Epstein-Barr virus. Allergol Immunopathol (Madr ). 2006;34:76-8.

4. Renn CN, Straff W, Dorfmuller A, Al Masaoudi T, Merk HF, Sachs B. Amoxicillin-induced exanthema in young adults with infectious mononucleosis: demonstration of drug-specific lymphocyte reactivity. $\mathrm{Br} J$ Dermatol. 2002;147:1166-70

5. Jappe U. Amoxicillin-induced exanthema in patients with infectious mononucleosis: allergy or transient immunostimulation? Allergy. 2007;62:1474-5.

6. Drug, Hypersensitivity. Basel, Freyburg: Karger; 2007.

7. Mardivirin L, Valeyrie-Allanore L, Branlant-Redon E, Beneton N, Jidar K, Barbaud A, et al. Amoxicillin-induced flare in patients with DRESS (Drug Reaction with Eosinophilia and Systemic Symptoms): report of seven cases and demonstration of a direct effect of amoxicillin on Human Herpesvirus 6 replication in vitro. Eur J Dermatol. 2010;20:68-73.

8. Pichler WJ, Tilch J. The lymphocyte transformation test in the diagnosis of drug hypersensitivity. Allergy. 2004;59:809-20.

9. Luque I, Leyva L, Jose TM, Rosal M, Mayorga C, Segura JM, et al. In vitro T-cell responses to beta-lactam drugs in immediate and nonimmediate allergic reactions. Allergy. 2001;56:611-8.

10. McCloskey GL, Massa MC. Cephalexin rash in infectious mononucleosis. Cutis. 1997:59:251-4.

11. Yoshimura T, Kurita $C$, Hayata M, Nagai H. Diagnosis of drug allergy by the lymphocyte stimulation test with the MTT [3-(4,5-dimethyl thiazol-2-yl)-2, 5-diphenyl tetrazolium bromide] assay. Biol Pharm Bull. 1993;16:686-9.

12. Romano A, Viola M, Bousquet PJ, Gaeta F, Valluzzi R, Caruso C, et al. A comparison of the performance of two penicillin reagent kits in the diagnosis of beta-lactam hypersensitivity. Allergy. 2007;62:53-8.

13. Romano A, Demoly P. Recent advances in the diagnosis of drug allergy. Curr Opin Allergy Clin Immunol. 2007;7:299-303.

14. Brockow K, Romano A, Blanca M, Ring J, Pichler W, Demoly P. General considerations for skin test procedures in the diagnosis of drug hypersensitivity. Allergy. 2002;57:45-51.

15. Lammintausta K, Kortekangas-Savolainen O. The usefulness of skin tests to prove drug hypersensitivity. Br J Dermatol. 2005;152:968-74.

16. Romano A, Viola M, Gaeta F, Rumi G, Maggioletti M. Patch testing in non-immediate drug eruptions. Allergy Asthma Clin Immunol. 2008;4:66-74.

17. Schissel DJ, Singer D, David-Bajar K. Azithromycin eruption in infectious mononucleosis: a proposed mechanism of interaction. Cutis. 2000;65:163-6.

18. Paily R. Quinolone drug rash in a patient with infectious mononucleosis. J Dermatol. 2000;27:405-6.

19. van der Linden $P D$, van der $L$, Vlug $A E$, Stricker BH. Skin reactions to antibacterial agents in general practice. J Clin Epidemiol. 1998;51:703-8.

20. Dakdouki GK, Obeid KH, Kanj SS. Azithromycin-induced rash in infectious mononucleosis. Scand J Infect Dis. 2002;34:939-41.

21. Timar L, Balo-Banga JM, Budai J. Infectious mononucleosis and drug exanthema. Orv Hetil. 1987;128:1871-4.

22. Copeman PW, Scrivener R. Amoxycillin rash. Br Med J. 1977;1:1354.

doi:10.1186/1710-1492-11-1

Cite this article as: Ónodi-Nagy et al:: Amoxicillin rash in patients with infectious mononucleosis: evidence of true drug sensitization. Allergy, Asthma \& Clinical Immunology 2015 11:1.

\section{Submit your next manuscript to BioMed Central and take full advantage of:}

- Convenient online submission

- Thorough peer review

- No space constraints or color figure charges

- Immediate publication on acceptance

- Inclusion in PubMed, CAS, Scopus and Google Scholar

- Research which is freely available for redistribution 